\title{
The Association Between Daily Physical Activity and Pain Among Patients with Knee Osteoarthritis: The Moderating Role of Pain Catastrophizing
}

\author{
Asimina Lazaridou, PhD, * Marc O. Martel, PhD, * Marise Cornelius, BA, * Olivia Franceschelli, BA,* \\ Claudia Campbell, PhD, ${ }^{\dagger}$ Michael Smith, PhD, ${ }^{\dagger}$ Jennifer A. Haythornthwaite, $\mathrm{PhD},{ }^{\dagger}$ John R. Wright, \\ MD, ${ }^{\ddagger}$ and Robert R. Edwards, PhD*
}

\begin{abstract}
Departments of *Anesthesiology, Perioperative, Perioperative \& Pain Medicine, Harvard Medical School, Brigham \& Women's Hospital, ${ }^{\ddagger}$ Orthopedic Surgery, Harvard Medical School, Brigham \& Women's Hospital, Chestnut Hill, Massachusetts; ${ }^{\dagger}$ Department of Psychiatry and Behavioral Sciences, Johns Hopkins University School of Medicine, Baltimore, Maryland, USA
\end{abstract}

Correspondence to: Robert R. Edwards, PhD, Brigham \& Women's Hospital, Pain Management Center, 850 Boylston St, Chestnut Hill, MA 02467, USA. Tel: 617-732-9486; Fax: 617-732-9050; E-mail: rredwards@partners.org.

Funding sources: This work was supported by National Institutes of Health grant R01 AG034982 (Edwards, PI). The funding body had no direct role in study design, data analysis, or the writing of the manuscript.

Conflicts of interest: We have no conflict of interest to declare.

\begin{abstract}
Objective. The primary objective of this study was to examine the day-to-day association between physical activity and pain intensity among a sample of patients with knee osteoarthritis (KOA) and the potential moderation of this association by negative cognitive processes. Methods. In this micro-longitudinal daily diary study, KOA patients $(\mathrm{N}=121)$ completed questionnaires assessing pain (Brief Pain Inventory) and psychosocial functioning (pain catrophizing scale, WOMAC McMaster Universities Osteoarthritis Index function, Patient-Reported Outcomes Measurement Information System [PROMIS; anxiety and depression], the Godin-Shephard Leisure-Time Physical Activity questionnaire, the six-minute walking test) and were then asked to report their levels of physical activity and pain intensity once per day for a period of seven days using an electronic diary. Results. Multilevel modeling analyses indicated that day-to-day increases in physical activity were associated with heightened levels of pain intensity $(B=$ $0.13 \mathrm{SE}=0.03, P<0.001)$. In addition, it was revealed that the association between physical activity and pain intensity was moderated by catastrophizing $(B=-0.01 \mathrm{SE}=0.002, P<0.05)$, with patients scoring higher in catastrophizing showing a relatively stronger link between day-to-day physical activity and increased knee pain. This effect was specific to catastrophizing, as depression and anxiety did not moderate the activity-pain relationship $(P>0.05)$. Conclusions. Our findings suggest that increases in daily physical activity are associated with concurrent increases in KOA patients' levels of knee pain, particularly among patients reporting higher levels of pain catastrophizing. These results may have clinical implications for the design and testing of interventions targeted at reducing catastrophizing and increasing physical activity among patients with chronic osteoarthritis pain.
\end{abstract}

Key Words: Catastrophizing; Physical Activity; Clinical Pain; Osteoarthritis

\section{Introduction}

Knee osteoarthritis (KOA) is among the most prevalent conditions causing persistent pain and disability and around the world [1-3]. The global cost of osteoarthritis $(\mathrm{OA})$ is high and includes substantial direct and indirect financial costs, significant physical limitations, reduced work productivity, and diminished health-related quality of life [4]. Patients who suffer from KOA often receive recommendations to increase their physical activity in order to improve physical functioning and prevent 
disability [5-7]. Moreover, a Cochrane report emphasized that effective patient-centered treatment should include local muscle strengthening and general aerobic fitness [8]. The authors of this review suggested that the evidence to support exercise was convincing and that the magnitude of the pain-reducing effect was equal to nonsteroidal anti-inflammatory medications. The American College of Sports Medicine (ACSM) and the American Heart Association (AHA) also suggest that people with arthritis should engage in 30 minutes or more of moderate-intensity aerobic physical activity every day to maintain their physical well-being [5, 9].

However, because of the symptoms associated with arthritis (e.g., joint swelling and stiffness, pain, and limitations in range of motion), exercise can often be painful for patients with OA [10-12]. Hence, despite the potential long-term benefits of physical activity for patients with chronic musculoskeletal pain, it is often challenging for KOA patients to regularly engage in exercise [13-15]. A recent review described substantial variability in the painfulness of exercise for patients with chronic musculoskeletal pain [16], and qualitative studies note that increased pain is one of the primary reasons for discontinuing physical activity and exercise programs [17]. This finding highlights the critical importance of understanding the patient-level factors that shape the association between activity and pain. One such factor is pain-related catastrophizing; our group and others have shown that increased levels of sensitivity to physical activity (defined as increases in pain during sustained standardized tasks such as time walking) are linked to heightened pain catastrophizing and increased disability $[11,18]$. Collectively, catastrophizing is one of the most important prospective predictors of pain outcomes in osteoarthritis [19-23].

To date, however, little research has directly evaluated the role of psychological factors in amplifying or attenuating the impact of physical activity on pain. Catastrophizing is hypothesized to contribute to clinical outcomes in chronic pain, in part by impacting health behaviors such as exercise [24]; specifically, the fear avoidance model suggests that catastrophizing about pain initiates a cycle of pain-related fear, impairment, and disability [25-28]. However, more recent research has demonstrated that pain can be context-specific. For example, environmental cues and competing goals can impact avoidance behaviors [29], highlighting the importance of determining the factors that contribute to daily fluctuations in joint pain among patients with KOA. Diary-based methodologies are commonly utilized approaches for evaluating such associations [30-32] and provide the opportunity to investigate links between cognitive/affective processes and daily pain outcomes. In this study, we aimed to investigate the day-to-day association between physical activity and pain intensity in KOA, and the potential psychosocial moderators (e.g., negative affect, catastrophizing) of this association. Our specific hypothesis was that catastrophizing would moderate the relationship between physical activity and daily knee pain.

\section{Methods}

One hundred twenty-one participants with chronic advanced (i.e., Kellgren-Lawrence grade 3+) KOA awaiting unilateral total knee replacement at Brigham \&Women's Hospital (Boston, MA, USA) were recruited and asked to complete self-report questionnaires including demographic psychosocial measures and pain-related scales. This analysis was part of a larger cohort study evaluating psychosocial variables predicting postsurgical pain after total knee replacement (TKR) surgery.

Following the study visit, patients were asked to report their levels of physical activity and pain intensity once a day for a period of seven days using numeric rating scales (NRS) on a handheld personal digital assistant (PDA). The involvement of human subjects in this study was reviewed, approved, and monitored by the Institutional Review Board (IRB) of Brigham \& Women's Hospital (Boston, MA, USA).

\section{Eligibility Criteria}

Patients were included if they met the following criteria: 1) age 50 years or older, 2) met the American College of Rheumatology criteria for knee OA, 3) scheduled to undergo total knee arthroplasty, 4) facility with the English language that is adequate to complete study procedures, 5) patients taking nonsteroidal anti-inflammatory drugs or acetaminophen must be on a stable dose one month before the study, 6) self-report of (current) pain $\geq 3(0-10$ scale). Patients were excluded for the following: 1) cognitive impairment preventing completion of study assessment procedures, 2) myocardial infarction within the past 12 months, 3) presence of Raynaud's phenomenon or severe neuropathy, 4) active vasculitis or severe peripheral vascular disease, 5) current infection, 6) use of oral steroids, 7) recent history of substance abuse or dependence, 8) confirmed diagnosis of periodic limb movement disorder or restless legs syndrome, 9) systemic inflammatory or autoimmune disorders such as rheumatoid arthritis and lupus, 10) severe anemia.

\section{Measures}

\section{Sociodemographic Data}

Sociodemographic information included date of birth; marital, educational, and current occupational status; duration of OA symptoms; medical comorbidities, etc.

\section{Catastrophizing}

The pain catastrophizing scale (PCS) [33] is a widely used self-report measure of catastrophic thinking associated with pain. The PCS has shown good psychometric properties in samples of chronic pain patients and controls $[34,35]$. The PCS is a 13 -item instrument that uses a five- 
point Likert scale ("not at all" to "all the time"), with higher scores indicating elevated levels of catastrophizing. The PCS examines three components of catastrophizing: rumination, magnification, and helplessness. Prior KOA studies have utilized a cutoff point of PCS $>16$ for identifying high catastrophizers in representative samples of KOA patients [23,36]. In our study, $37.8 \%$ of patients were above this cut-point, though the present analyses utilize PCS scores as a continuous variable.

\section{WOMAC McMaster Universities Osteoarthritis Index}

\section{Function}

The WOMAC is a self-administered health status measure that assesses the dimensions of pain, stiffness, and function (either separately or as an overall index) in patients with OA of the hip or knee. Each dimension contains multiple items rated on 100-mm visual analog scale (VAS). For the current study, we utilized the WOMAC-function subscale, which contains items relating to walking, climbing stairs, and performing activities of daily living. Scores range from 0 to 96 for the total WOMAC, where 0 represents the best health status and 96 the worst possible status. The WOMAC has been shown to have good psychometric properties including good test-retest reliability, internal consistency, and responsiveness in OA patients undergoing total knee or hip arthroplasty [37]. The range of WOMAC scores from this sample is broadly similar to other KOA studies [23].

\section{The Godin-Shephard Leisure-Time Physical Activity Questionnaire}

The Godin-Shephard Leisure-Time Physical Activity Questionnaire (GSLTPAQ) [38] was developed to classify physical activities/exercises performed by adults and to determine their activity levels. Activities are classified into three subgroups, including "strenuous," "moderate," and "light," in the Leisure-Time Exercise Questionnaire, which is practical and easy to fill out.

\section{The Six-Minute Walking Test}

In the six-minute walking test $(6 \mathrm{MW})$ test, subjects are asked to cover as much distance as possible in six minutes while walking laps on a standardized indoor course [11].

\section{Emotional Distress-Anxiety and Depression}

Participants completed the Patient-Reported Outcomes Measurement Information System (PROMIS) anxiety and depression short forms, which are widely used and extensively validated $[39,40]$. The anxiety subscale consists of seven items that ask respondents about the frequency with which they have experienced emotions such as fear, stress, and anxiety ("never" to "always"). The depression subscale consists of eight items in which respondents indicate the frequency with which they have experienced emotions such as worthlessness, hopelessness, and sadness ("never" to "always"). Higher scores indicate more severe symptoms of emotional distress.

\section{Pain Intensity}

Patients were asked to report their mean levels of daily knee pain intensity (0 "no pain" to 100 "pain as bad as it could be") once per day for a period of seven days using numeric rating scales (NRS) on a personal digital assistant (PDA, Palm Tungsten E2).

\section{Physical Activity}

Patients were asked to report their levels of physical activity (0 "not at all active" to 100 "extremely active") once a day for a period of seven days using a VAS on a PDA. The VAS was scored from 0 to 100; similar patient-reported global physical activity scores have often been used in pain research [41] and other research assessing physical functioning [42-44]. Reviews of the literature on assessment of physical activity in OA patients note that a number of single-item numeric scales for measuring global physical activity have been validated $[45,46]$. The item used here is most similar to the patient VAS for physical activity $[45,46]$, but it was modified to refer to daily physical activity.

Participants completed the electronic daily diary surveys once per day for seven days; participants completed surveys in the evening and provided ratings (i.e., pain, physical activity) for the preceding day (e.g., How physically active were you today?).

\section{Data Analysis}

All analyses were conducted using IBM-SPSS v.21. Descriptive data for continuous variables are presented as means and standard deviations, and data for categorical variables are presented as percentages. Primary analyses were conducted using multilevel modeling due to the hierarchical data structure of the present study, in which repeated measures (level 1 units) were nested within participants (level 2 units). Given that multilevel modeling can account for an unbalanced data structure and/or random missing data $[47,48]$, all participants could be included in multilevel analyses without using any data imputation procedure. All multilevel models were built using daily pain intensity as the dependent (i.e., outcome) variable. Given that we were interested in examining the time course of pain intensity responses, we built a multilevel model in which "physical activity" was first included as a level 1 independent variable. Level 2 catastrophizing (i.e., PCS) and negative affect (PROMISanxiety and PROMIS-depression) scores were then added to the model, which permitted examination of the effects of physical activity and psychosocial factors on pain responses. In order to examine whether any relationship between daily physical activity and daily pain intensity was influenced by psychosocial factors, two-way (e.g., 
level 2 catastrophizing * level 1 physical activity) interaction terms were then specified and included in the model.

\section{Results}

\section{Descriptive Statistics}

Descriptive statistics for study measures are presented in Table 1 for OA patients. The average age of patients (SD, range) was $66.3(8.5,50-87)$ years. In this sample, $58.9 \%$ of patients were women and $83.9 \%$ were white. Scores on pain, physical activity, and psychological (i.e., PCS) measures are comparable to those that have been observed in previous studies among patients with chronic pain [49-51]. In terms of missing values, analyses were also conducted to examine the amount of missing data for each of the main independent variables (catastrophizing, physical activity) and for the study outcome (pain intensity). In our study, the amount of missing data was very low (i.e., $2 \%$ of diary entries were missing).

Before conducting primary analyses, the potential confounding influence of patient demographics (i.e., age, sex, ethnicity) or negative affect (i.e., anxiety, depression) on pain responses was examined. Given that none of these variables was significantly associated with pain scores (all $P>0.05$ ), they were not included as covariates in the multilevel models reported below. In addition, we ran Pearson correlation analysis to explore the relationship between PCS, pain intensity, and physical activity (weekly average). PCS was significantly correlated with physical activity and pain intensity $(P<0.05)$.

We explored the relationship between WOMACfunction/disability, pain catastrophizing, PROMISdepression, PROMIS-anxiety, and pain catastrophizing (Table 2). WOMAC-function/disability was significantly correlated with pain catastrophizing and PROMISanxiety $(P<0.05)$.

In addition, we explored the relationship between the GSLTPAQ, the $6 \mathrm{MW}$, and pain catastrophizing.

Catastrophizing was significantly negatively correlated with the GSLTPAQ $(P<0.05)$ and the $6 \mathrm{MW}$ $(P<0.05)$ (Table 2$)$.

\section{Association Between Catastrophizing and Pain}

Results from multilevel modeling (MLM) analyses revealed a significant main effect of PCS scores, indicating that higher levels of catastrophizing were associated with elevated pain responses $(B=0.64, S E=0.16$, $P<0.001$ ).

\section{Day-to-Day Association Between Physical Activity and Pain}

Results from MLM analyses revealed a significant main effect of physical activity, indicating that increases in physical activity by patients were associated with elevated pain responses $(\mathrm{B}=0.13, \mathrm{SE}=0.03, P<0.001)$.
Table 1. Sample characteristics and descriptive data for main variables

\begin{tabular}{lc}
\hline Ethnicity, \% white & 83.9 \\
Sex, \% women & 58.9 \\
Age, y & $66.3 \pm 8.5$ \\
BMI, kg/m ${ }^{2}$ & $30.82 \pm 6.31$ \\
WOMAC (disability) & $43.19 \pm 21.0$ \\
PROMIS-anxiety & $42.6 \pm 79.7$ \\
PROMIS-depression & $37.0 \pm 79.6$ \\
Catastrophizing (PCS) & $14.4 \pm 12.3$ \\
Average daily pain intensity (0-100) & $39.1 \pm 20.5$ \\
Average daily physical activity (0-100) & $44.3 \pm 21.3$ \\
Godin-Shepard Leisure-Time Physical Activity & $26.3 \pm 30.8$ \\
Six-minute walking test distance, feet & $915.93 \pm 266.53$ \\
\hline
\end{tabular}

Average daily pain intensity and physical activity are aggregated scores across the seven-day diary period, \pm SD.

$\mathrm{BMI}=$ body mass index; GSLTPAQ $=$ The Godin-Shephard Leisure-Time Physical Activity Questionnaire; PCS = pain catastrophizing scale; PROMIS $=$ Patient Reported Outcomes Measurement Information System (reported as T-scores); WOMAC $=$ Western Ontario \& McMaster Universities Osteoarthritis Index.

\section{Moderating Role of Catastrophizing (Catastrophizing-Physical Activity Interaction)}

Results of a subsequent multilevel moderation analysis revealed that the relationship between physical activity and pain intensity was moderated by catastrophizing $(\mathrm{B}=-0.01, \mathrm{SE}=0.002, P<0.001)$ (Figure 1$)$. More specifically, results indicated that increases in pain on days of heightened physical activity were more pronounced among patients with relatively higher levels of catastrophizing (Table 3). Patients who are relatively low in catastrophizing reported an average daily pain intensity score of 31.3 (on the 0-100 scale) on low-physical activity days and an average daily pain intensity of 38.8 on high-physical activity days. This represents a $24 \%$ increase in pain intensity on days with high (relative to low) physical activity for low catastrophizers. In contrast, patients who are relatively high in catastrophizing report an average daily pain intensity of 30.5 on lowphysical activity days and an average daily pain intensity of 60.5 on high-physical activity days, representing a $98 \%$ increase in pain intensity.

In addition, sensitivity analyses indicated that the association between physical activity and pain was not moderated by any other demographic (i.e., age, sex, ethnicity) or psychological (i.e., anxiety, depression) variables (all $P>0.05)$.

\section{Discussion}

As expected, we found that day-to-day increases in physical activity were associated with heightened levels of pain intensity among patients with knee pain; this is consistent with findings from previous KOA studies [52-57]. Most interestingly, activity-related increases in KOA pain were more pronounced among patients with high levels of catastrophizing. These results are consistent with previous work linking activity-related pain with catastrophizing 
Table 2. Pearson correlations between pain catastrophizing (anxiety, depression) and WOMAC, six-minute test, and Godin-Shepard Leisure-Time Physical Activity

\begin{tabular}{|c|c|c|c|c|c|c|}
\hline & 1. & 2. & 3. & 4. & 5. & 6. \\
\hline 1. Godin-Shepard Leisure-Time Physical Activity & 1 & & & & & \\
\hline 2. Pain catastrophizing (PCS) & $-0.14^{*}$ & 1 & & & & \\
\hline 3. WOMAC (disability) & $-0.16^{*}$ & $0.52 * *$ & 1 & & & \\
\hline 4. PROMIS-anxiety & 0.07 & $0.40^{* *}$ & $0.28 * *$ & 1 & & \\
\hline 5. PROMIS-depression & $-0.18^{*}$ & $0.42 * *$ & $0.25^{* *}$ & $0.64 * *$ & 1 & \\
\hline 6. Six-minute walking test & $0.36^{* *}$ & $-0.21 *$ & $-0.21 *$ & -0.08 & $-0.16^{*}$ & 1 \\
\hline
\end{tabular}

PCS = pain catastrophizing scale; PROMIS = Patient Reported Outcomes Measurement Information System; WOMAC = Western Ontario \& McMaster Universities Osteoarthritis Index.

$* P<0.05 ; * P<0.01$.

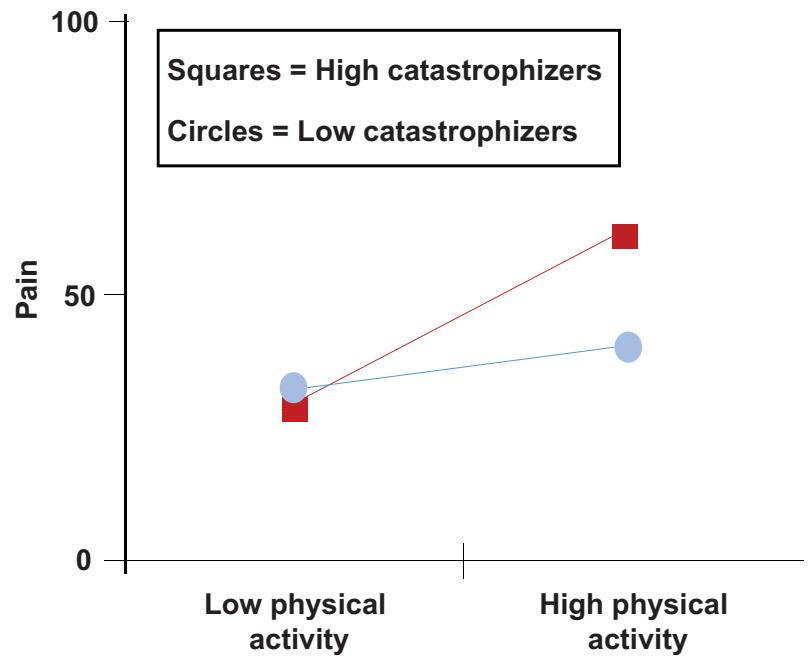

Figure 1. Interaction effects between daily pain intensity, physical exercise, and catastrophizing.

Table 3. Multilevel model examining the moderating role of catastrophizing in the association between physical activity and pain intensity

\begin{tabular}{lllll}
\hline Fixed Effects & $\beta$ & $\mathrm{SE}$ & $\mathrm{t}$ & $P$ Value \\
\hline $\begin{array}{l}\text { Intercept } \\
\text { Level 2 }\end{array} \quad 42.7$ & 1.9 & 21.4 & $<.001$ \\
$\quad \begin{array}{l}\text { Catastrophizing } \\
\text { Level 1 }\end{array}$ & 0.64 & 0.16 & 3.9 & $<0.001$ \\
$\quad \begin{array}{l}\text { Physical activity } \\
\text { Level 2 } \times \text { level 1 } \\
\quad \text { Catastrophizing } \times \text { physical activity }\end{array}$ & -0.01 & 0.002 & -2.2 & $<0.05$
\end{tabular}

Values are from the final model.

$\beta=$ unstandardized regression coefficient.

among patients with KOA [11]. In addition, catastrophizing's moderating role is consistent with other psychosocial research suggesting that catastrophizing can impact an array of function-related processes in patients with arthritis and other chronic musculoskeletal conditions [5862]. For example, catastrophic thinking can lead to more unhealthy behaviors and limit response choices, such as when catastrophic thoughts lead to exaggerated emotional responses, which can lead to avoidance behaviors. We also observed that catastrophizing was associated with reduced physical functioning on measures such as the GSLTPAQ, which assesses daily exercise, the 6MW, a measure of functional walking ability, and the WOMAC, which measures patients' perceived ability to engage in walking, stair-climbing, housework, and other daily physical tasks. Collectively, these results indicate that catastrophizing may exert short-term effects (i.e., increases in pain intensity) on activity-related pain and longer-term effects on KOA patients' degree of physical disability.

Our findings suggest that engaging in physical activity is likely to represent a special challenge for KOA patients who score high in catastrophizing. High catastrophizing has been shown in previous studies to be associated with lower rates of physical activity [63-67] and a greater tendency to engage in sedentary behaviors such as bed rest [68]. It is possible that these patients might be more likely to avoid physical activity because of concerns that activity could exacerbate their pain symptoms and contribute to worsening disease severity [20,69-71]. In addition, catastrophizing is associated with greater perceptions of difficulty in completing movements and activities [72]. Such perceptions may not be entirely unwarranted, as recent studies have shown that catastrophizing and fear avoidance beliefs are correlated with postural instability in OA patients [20] and with maladaptive patterns of muscle activation in other conditions such as chronic low back pain [73,74]. In addition, catastrophizing does appear to adversely affect performance in standardized tests of physical capacity in people with chronic musculoskeletal conditions such as arthritis, back pain, and fibromyalgia $[66,75-78]$.

Our findings agree with other studies suggesting that OA patients who are relatively high in catastrophizing may be particularly susceptible to experiencing flare-ups over the course of physical therapy or other types of activity-based interventions $[70,79,80]$. One potential mechanism contributing to this effect may be decrements in exercise-induced hypoalgesia in patients high in catastrophizing. Exercise is consistently associated with acute reductions in pain sensitivity in healthy adults [81-83], and deficiencies in these endogenous inhibitory pathways are thought to contribute to the development and 
maintenance of some chronic pain conditions $[84,85]$. Several recent studies have shown that high pain catastrophizing predicts deficits in exercise-associated hypoalgesia $[79,86]$, which may contribute to our observations of larger activity-provoked increases in knee pain among patients with high PCS scores.

Collectively, these findings suggest that minimizing patients' catastrophic thoughts might contribute to attenuating physical activity-related pain flares and augment the overall impact of activity-based treatment programs. Given that increased physical activity, exercise, weight loss, and physical therapy are commonly recommended treatments, interventions that promote compliance with such treatments could have substantial and widespread benefits for KOA patients $[6,8,87]$. Studies on chronic pain management indicate that functional activities tailored to individual needs might be more effective for improving pain and disability $[88,89]$ than general recommendations on physical activity, and such tailoring should take into account psychosocial factors such as catastrophizing. Overall, the findings of this study have implications for understanding the psychological mechanisms underlying physical activity behaviors in the context of pain. Some researchers have suggested that anxiety and depression are associated with decreased levels of physical activity; however, the role of pain-negative cognitions (e.g., catastrophizing) is not well studied. This study offers a framework on the potential mechanisms affecting sedentary behaviors in patients with OA pain. For example, it seems that negative cognitions on pain lead to less physical activity, which as a result can lead to sedentary patterns of behaviors and unhealthy habits in patients with OA. We hope that these findings will stimulate further investigation into the interaction of psychological and physiological outcomes in the context of chronic pain and assist in the design of future physical activity-based interventions.

However, the limitations of this study should be considered when interpreting these results. For instance, we assessed only subjective self-reported physical activity in the present study. Prior work in samples of chronic pain patients suggests that there is suboptimal agreement between subjective physical function and objective physical function in patients with chronic pain, and the magnitude of such discrepancies is related to negative cognitive factors such as catastrophizing [64]. We did observe that catastrophizing was associated with reduced performance on an objectively measured standardized walking task; future studies in this area may benefit from the use of combined self-report and objective accelerometerbased data when assessing physical activity [90]. Another study limitation is that we recruited patients awaiting joint replacement, reflecting a population with advanced OA that might not be representative of all OA patients. However, prior community-based studies of KOA samples with less advanced disease have shown that patients report similar levels of catastrophizing [23]. In addition, our sample does have substantial variability in the functional impact of OA, and controlling for physical functioning scores (e.g., WOMAC) in our analyses did not alter the findings, suggesting that the amplifying effect of catastrophizing on activity-associated pain is observed even in patients with less functionally impairing symptoms. Finally, we do not have physiological mechanismbased data (e.g., information regarding posture, gait, muscle strength, or exercise-induced hypoalgesia), which might illuminate some of the pathways by which the observed associations unfold. Despite these limitations, however, the current results are encouraging, and we hope that they will stimulate further investigation into the links between psychological factors and physical activity in shaping the experience of chronic pain.

\section{References}

1. Losina E, Paltiel AD, Weinstein AM, et al. Lifetime medical costs of knee osteoarthritis management in the United States: Impact of extending indications for total knee arthroplasty. Arthritis Care Res (Hoboken) 2015;67(2):203-15.

2. Smith E, Hoy DG, Cross M, et al. The global burden of other musculoskeletal disorders: Estimates from the Global Burden of Disease 2010 study. Ann Rheum Dis 2014;73(8):1462-9.

3. Cross M, Smith E, Hoy D, et al. The global burden of hip and knee osteoarthritis: Estimates from the global burden of disease 2010 study. Ann Rheum Dis 2014; 73(7):1323-30.

4. Altman RD. Early management of osteoarthritis. Am J Manag Care 2010;16(suppl management):S41-7.

5. Nelson ME, Rejeski WJ, Blair SN, et al. Physical activity and public health in older adults: Recommendation from the American College of Sports Medicine and the American Heart Association. Med Sci Sports Exerc 2007;39 (8):1435-45.

6. Alshami AM. Knee osteoarthritis related pain: A narrative review of diagnosis and treatment. Int $\mathrm{J}$ Health Sci (Qassim) 2014;8(1):85-104.

7. Kelley GA, Kelley KS, Hootman JM, Jones DL. Effects of community-deliverable exercise on pain and physical function in adults with arthritis and other rheumatic diseases: A meta-analysis. Arthritis Care Res (Hoboken) 2011;63(1):79-93.

8. Fransen M, McConnell S, Harmer AR, et al. Exercise for osteoarthritis of the knee: A Cochrane systematic review. Br J Sports Med 2015;49(24):1554-7.

9. Haskell WL, Lee IM, Pate RR, et al. Physical activity and public health: Updated recommendation for adults from the American College of Sports Medicine and the American Heart Association. Circulation 2007;116(9):1081-93.

10. Baxter SV, Hale LA, Stebbings S, et al. Walking is a feasible physical activity for people with rheumatoid 
arthritis: A feasibility randomized controlled trial. Musculoskeletal Care 2016;14(1):47-56.

11. Wideman TH, Finan PH, Edwards RR, et al. Increased sensitivity to physical activity among individuals with knee osteoarthritis: Relation to pain outcomes, psychological factors, and responses to quantitative sensory testing. Pain 2014;155 (4):703-11.

12. Cruz-Almeida Y, King CD, Goodin BR, et al. Psychological profiles and pain characteristics of older adults with knee osteoarthritis. Arthritis Care Res (Hoboken) 2013;65(11):1786-94.

13. Harden RN, Wallach G, Gagnon CM, et al. The osteoarthritis knee model: Psychophysical characteristics and putative outcomes. J Pain 2013;14(3):281-9.

14. Wilcox S, Der Ananian C, Abbott J, et al. Perceived exercise barriers, enablers, and benefits among exercising and nonexercising adults with arthritis: Results from a qualitative study. Arthritis Rheum 2006;55(4):616-27.

15. Rosemann T, Laux G, Szecsenyi J, Wensing M, Grol R. Pain and osteoarthritis in primary care: Factors associated with pain perception in a sample of 1,021 patients. Pain Med 2008;9(7):903-10.

16. Smith BE, Hendrick P, Smith TO, et al. Should exercises be painful in the management of chronic musculoskeletal pain? A systematic review and metaanalysis. Br J Sports Med. 51(23):1679-1687.

17. Slade SC, Patel S, Underwood M, Keating JL. What are patient beliefs and perceptions about exercise for nonspecific chronic low back pain? A systematic review of qualitative studies. Clin J Pain 2014;30 (11):995-1005.

18. Sullivan MJ, Lariviere C, Simmonds M. Activity-related summation of pain and functional disability in patients with whiplash injuries. Pain 2010;151 (2):440-6

19. Alschuler KN, Molton IR, Jensen MP, Riddle DL. Prognostic value of coping strategies in a communitybased sample of persons with chronic symptomatic knee osteoarthritis. Pain 2013;154(12):2775-81.

20. Sanchez-Heran A, Agudo-Carmona D, Ferrer-Pena $\mathrm{R}$, et al. Postural stability in osteoarthritis of the knee and hip: Analysis of association with pain catastrophizing and fear-avoidance beliefs. PM R 2016;8 (7):618-28.

21. Sturgeon JA, Zautra AJ, Arewasikporn A. A multilevel structural equation modeling analysis of vulnerabilities and resilience resources influencing affective adaptation to chronic pain. Pain 2014;155(2):292-8.

22. Edwards RR, Calahan C, Mensing G, Smith M, Haythornthwaite JA. Pain, catastrophizing, and depression in the rheumatic diseases. Nat Rev Rheumatol 2011;7(4):216-24.

23. Riddle DL, Wade JB, Jiranek WA, Kong X. Preoperative pain catastrophizing predicts pain outcome after knee arthroplasty. Clin Orthop Relat Res 2010;468(3):798-806.

24. Edwards RR, Cahalan C, Calahan C, et al. Pain, catastrophizing, and depression in the rheumatic diseases. Nat Rev Rheumatol 2011;7(4):216-24.

25. Vlaeyen JW, Linton SJ. Fear-avoidance and its consequences in chronic musculoskeletal pain: A state of the art. Pain 2000;85(3):317-32.

26. Vlaeyen JW, Linton SJ. Fear-avoidance model of chronic musculoskeletal pain: 12 years on. Pain 2012; 153(6):1144-7.

27. Wideman TH, Asmundson GG, Smeets RJ, et al. Rethinking the fear avoidance model: Toward a multidimensional framework of pain-related disability. Pain 2013;154(11):2262-5.

28. Crombez G, Eccleston C, Van Damme S, Vlaeyen JW, Karoly P. Fear-avoidance model of chronic pain: The next generation. Clin J Pain 2012;28(6):475-83.

29. Vlaeyen JW, Crombez G, Linton SJ. The fearavoidance model of pain. Pain 2016;157(8):1588-9.

30. Schneider S, Junghaenel DU, Keefe FJ, et al. Individual differences in the day-to-day variability of pain, fatigue, and well-being in patients with rheumatic disease: Associations with psychological variables. Pain 2012;153(4):813-22.

31. Marceau LD, Link C, Jamison RN, Carolan S. Electronic diaries as a tool to improve pain management: Is there any evidence? Pain Med 2007;8(suppl 3):S101-9.

32. Davis MC, Zautra AJ, Wolf LD, Tennen H, Yeung EW. Mindfulness and cognitive-behavioral interventions for chronic pain: Differential effects on daily pain reactivity and stress reactivity. J Consult Clin Psychol 2015;83(1):24-35.

33. Sullivan MJ, Bishop SR, Pivik J. The pain catastrophizing scale: Development and validation. Psychol Assess 1995;7(4):524-32.

34. Pavlin DJ, Sullivan MJ, Freund PR, Roesen K. Catastrophizing: A risk factor for postsurgical pain. Clin J Pain 2005;21(1):83-90.

35. Osman A, Barrios FX, Kopper BA, et al. Factor structure, reliability, and validity of the pain catastrophizing scale. J Behav Med 1997;20(6):589-605.

36. Lecorney J, Verhoeven F, Chouk M, et al. Correlation between catastrophizing and Lequesne index in case of osteoarthritis of the knee: A prospective study. Joint Bone Spine. doi: 10.1016/j.jbspin.2017.10.002. [Epub ahead of print].

37. Wolfe F. Determinants of WOMAC function, pain and stiffness scores: Evidence for the role of low back pain, symptom counts, fatigue and depression in osteoarthritis, rheumatoid arthritis and fibromyalgia. Rheumatology (Oxford) 1999;38(4):355-61.

38. Godin G, Shephard RJ. A simple method to assess exercise behavior in the community. Can J Appl Sport Sci 1985;10(3):141-6. 
39. Pilkonis PA, Choi SW, Salsman JM, et al. Assessment of self-reported negative affect in the NIH Toolbox. Psychiatry Res 2013;206(1):88-97.

40. Cella D, Riley W, Stone A, et al. The PatientReported Outcomes Measurement Information System (PROMIS) developed and tested its first wave of adult self-reported health outcome item banks: 2005-2008. J Clin Epidemiol 2010;63(11):1179-94.

41. Williams K, Frei A, Vetsch A, et al. Patient-reported physical activity questionnaires: A systematic review of content and format. Health Qual Life Outcomes 2012;10(1):28.

42. Gardiner PA, Reid N, Gebel K, Ding D. Sitting time and physical function in australian retirees: An analysis of bidirectional relationships. J Gerontol A Biol Sci Med Sci. doi: 10.1093/gerona/gly008. [Epub ahead of print].

43. Jakobsen MD, Sundstrup E, Brandt M, Andersen LL. Psychosocial benefits of workplace physical exercise: Cluster randomized controlled trial. BMC Public Health 2017;17(1):798.

44. Klinger R, Kothe R, Schmitz J, Kamping S, Flor H. Placebo effects of a sham opioid solution: A randomized controlled study in patients with chronic low back pain. Pain 2017;158(10):1893-902.

45. Naal FD, Impellizzeri FM, Leunig M. Which is the best activity rating scale for patients undergoing total joint arthroplasty? Clin Orthop Relat Res 2009;467 (4):958-65.

46. Terwee CB, Bouwmeester W, van Elsland SL, de Vet HC, Dekker J. Instruments to assess physical activity in patients with osteoarthritis of the hip or knee: A systematic review of measurement properties. Osteoarthritis Cartilage 2011;19(6):620-33.

47. Peugh JL. A practical guide to multilevel modeling. J Sch Psychol 2010;48(1):85-112.

48. Singer JD, Willett JB. Applied Longitudinal Data Analysis: Modeling Change and Event Occurrence. Oxford: Oxford University Press; 2003.

49. Brown CA, El-Deredy W, Jones AK. When the brain expects pain: Common neural responses to pain anticipation are related to clinical pain and distress in fibromyalgia and osteoarthritis. Eur J Neurosci 2014; 39(4):663-72.

50. Brummett CM, Urquhart AG, Hassett AL, et al. Characteristics of fibromyalgia independently predict poorer long-term analgesic outcomes following total knee and hip arthroplasty. Arthritis Rheumatol 2015; 67(5):1386-94.

51. Martel MO, Finan PH, Dolman AJ, et al. Self-reports of medication side effects and pain-related activity interference in patients with chronic pain: A longitudinal cohort study. Pain 2015;156(6):1-100.

52. Fukutani N, Iijima H, Aoyama T, et al. Knee pain during activities of daily living and its relationship with physical activity in patients with early and severe knee osteoarthritis. Clin Rheumatol 2016;35 (9):2307-16.

53. Murphy SL, Schepens Niemiec S, Lyden AK, Kratz AL. Pain, fatigue, and physical activity in osteoarthritis: The moderating effects of pain- and fatiguerelated activity interference. Arch Phys Med Rehabil 2016;97(9):S201-9.

54. Murphy SL, Lyden AK, Kratz AL, et al. Characterizing pain flares from the perspective of individuals with symptomatic knee osteoarthritis. Arthritis Care Res (Hoboken) 2015;67(8):1103-11.

55. Paxton RJ, Melanson EL, Stevens-Lapsley JE, Christiansen CL. Physical activity after total knee arthroplasty: A critical review. World J Orthop 2015; 6(8):614-22.

56. Thomas SG, Pagura SM, Kennedy D. Physical activity and its relationship to physical performance in patients with end stage knee osteoarthritis. J Orthop Sports Phys Ther 2003;33(12):745-54.

57. Creamer P, Lethbridge-Cejku M, Hochberg MC. Where does it hurt? Pain localization in osteoarthritis of the knee. Osteoarthritis Cartilage 1998;6 (5):318-23.

58. Campbell CM, Buenaver LF, Finan P, et al. Sleep, pain catastrophizing, and central sensitization in knee osteoarthritis patients with and without insomnia. Arthritis Care Res (Hoboken) 2015;67(10):1387-96.

59. Edwards RR, Giles J, Bingham CO 3rd, et al. Moderators of the negative effects of catastrophizing in arthritis. Pain Med 2010;11(4):591-9.

60. Edwards RR, Haythornthwaite JA, Smith MT, Klick B, Katz JN. Catastrophizing and depressive symptoms as prospective predictors of outcomes following total knee replacement. Pain Res Manag 2009;14 (4):307-11.

61. Turk DC, Fillingim RB, Ohrbach R, Patel KV. Assessment of psychosocial and functional impact of chronic pain. J Pain 2016;17(suppl 9):T21-49.

62. Yamada K, Matsudaira K, Imano H, Kitamura A, Iso $\mathrm{H}$. Influence of work-related psychosocial factors on the prevalence of chronic pain and quality of life in patients with chronic pain. BMJ Open 2016;6(4):e010356.

63. Bousema EJ, Verbunt JA, Seelen HA, Vlaeyen JW, Knottnerus JA. Disuse and physical deconditioning in the first year after the onset of back pain. Pain 2007; 130(3):279-86.

64. Estevez-Lopez F, Alvarez-Gallardo IC, SeguraJimenez V, et al. The discordance between subjectively and objectively measured physical function in women with fibromyalgia: Association with catastrophizing and self-efficacy cognitions. The AlAndalus Project. Disabil Rehabil 2016;40(3): 329-337.

65. Vincent HK, George SZ, Seay AN, Vincent KR, Hurley RW. Resistance exercise, disability, and pain catastrophizing in obese adults with back pain. Med Sci Sports Exerc 2014;46(9):1693-701. 
66. Nijs J, Van de Putte K, Louckx F, Truijen S, De Meirleir K. Exercise performance and chronic pain in chronic fatigue syndrome: The role of pain catastrophizing. Pain Med 2008;9(8):1164-72.

67. Chiarotto A, Fortunato S, Falla D. Predictors of outcome following a short multimodal rehabilitation program for patients with whiplash associated disorders. Eur J Phys Rehabil Med 2015;51(2):133-41.

68. Verbunt JA, Sieben J, Vlaeyen JWS, Portegijs P, André Knottnerus J. A new episode of low back pain: Who relies on bed rest? Eur J Pain 2008;12(4):508-16.

69. Sullivan M, Tanzer M, Stanish W, et al. Psychological determinants of problematic outcomes following total knee arthroplasty. Pain 2009;143(1-2):123-9.

70. Witvrouw E, Pattyn E, Almqvist KF, et al. Catastrophic thinking about pain as a predictor of length of hospital stay after total knee arthroplasty: A prospective study. Knee Surg Sports Traumatol Arthrosc 2009;17(10):1189-94.

71. Somers TJ, Keefe FJ, Pells JJ, et al. Pain catastrophizing and pain-related fear in osteoarthritis patients: Relationships to pain and disability. J Pain Symptom Manage 2009;37(5):863-72.

72. Perez-Fernandez M, Lerma-Lara S, Ferrer-Pena R, et al. Fear and difficulty perceived when visualizing therapeutic exercise in patients with chronic low back pain: A cross-sectional study. J Exerc Rehabil 2015; 11(6):345-55.

73. Pakzad M, Fung J, Preuss R. Pain catastrophizing and trunk muscle activation during walking in patients with chronic low back pain. Gait Posture 2016;49: 73-7.

74. Hulst M, Vollenbroek-Hutten MM, Schreurs KM, Rietman JS, Hermens HJ. Relationships between coping strategies and lumbar muscle activity in subjects with chronic low back pain. Eur J Pain 2010;14 (6):640-7.

75. Marcum ZA, Zhan HL, Perera S, et al. Correlates of gait speed in advanced knee osteoarthritis. Pain Med 2014;15(8):1334-42.

76. Nijs J, Meeus M, Heins M, et al. Kinesiophobia, catastrophizing and anticipated symptoms before stair climbing in chronic fatigue syndrome: An experimental study. Disabil Rehabil 2012;34(15):1299-305.

77. Lariviere C, Bilodeau M, Forget R, Vadeboncoeur R, Mecheri H. Poor back muscle endurance is related to pain catastrophizing in patients with chronic low back pain. Spine (Phila Pa 1976) 2010;35 (22):E1178-86.
78. Soriano-Maldonado A, Ruiz JR, Aparicio VA, et al. Association of physical fitness with pain in women with fibromyalgia: The Al-Andalus Project. Arthritis Care Res (Hoboken) 2015;67(11):1561-70.

79. Brellenthin AG, Crombie KM, Cook DB, Sehgal N, Koltyn KF. Psychosocial influences on exerciseinduced hypoalgesia. Pain Med 2017;18(3):538-50.

80. Shelby RA, Somers TJ, Keefe FJ, et al. Domain specific self-efficacy mediates the impact of pain catastrophizing on pain and disability in overweight and obese osteoarthritis patients. J Pain 2008;9 (10):912-9.

81. Stagg NJ, Mata HP, Ibrahim MM, et al. Regular exercise reverses sensory hypersensitivity in a rat neuropathic pain model: Role of endogenous opioids. Anesthesiology 2011;114(4):940-8.

82. Koltyn KF. Analgesia following exercise: A review. Sports Med 2000;29(2):85-98.

83. Vaegter HB, Handberg G, Graven-Nielsen T. Similarities between exercise-induced hypoalgesia and conditioned pain modulation in humans. Pain 2014;155(1):158-67.

84. Vaegter HB, Handberg G, Graven-Nielsen T, Edwards R. Hypoalgesia after exericse and cold pressor test are reduced in chronic musculuskeletal pain patients with high pain sensitivity. Clin J Pain 2016: 32(1):58-69.

85. Staud R, Robinson ME, Price DD. Isometric exercise has opposite effects on central pain mechanisms in fibromyalgia patients compared to normal controls. Pain 2005;118(1-2):176-84.

86. Naugle KM, Naugle KE, Fillingim RB, Riley JL 3rd. Isometric exercise as a test of pain modulation: Effects of experimental pain test, psychological variables, and sex. Pain Med 2014;15(4):692-701.

87. Bijlsma JW, Knahr K. Strategies for the prevention and management of osteoarthritis of the hip and knee. Best Pract Res Clin Rheumatol 2007;21 (1):59-76.

88. Liddle SD, Gracey JH, Baxter GD. Advice for the management of low back pain: A systematic review of randomised controlled trials. Man Ther 2007;12 (4):310-27.

89. Ambrose KR, Golightly YM. Physical exercise as non-pharmacological treatment of chronic pain: Why and when. Best Pract Res Clin Rheumatol 2015;29 (1):120-30.

90. Wilson AC, Palermo TM. Physical activity and function in adolescents with chronic pain: A controlled study using actigraphy. J Pain 2012;13(2):121-30. 